\title{
Indicadores demográficos y estimación de la población de canes en el distrito de Bellavista, Callao - Perú
}

\author{
Demography and dog population estimation in Bellavista district, Callao - Peru \\ César Harada ${ }^{1}$, Daphne León ${ }^{1}$, Norma Gamarra², Néstor Falcón ${ }^{1}$ \\ RESUMEN
}

El estudio tuvo como objetivo estimar indicadores demográficos y la población de canes con dueño en el distrito de Bellavista, Callao. Se utilizó una encuesta como herramienta de recolección de información, la que fue aplicada en las viviendas del distrito, las que fueron seleccionadas a partir de un muestreo bietápico. Se seleccionó una muestra del 20\% de las manzanas de cada zona, siguiendo un muestreo sistemático. Asimismo, en cada zona se seleccionaron 10 viviendas a encuestar. Las características demográficas de la población de canes se resumieron mediante estadística descriptiva. Se encontró que el 56,1\% de hogares poseían al menos un can. El promedio de canes, entre las viviendas que tenían canes, fue de 1,6. Se encontró una relación humano: can de 5,6: 1. La edad promedio de los canes fue de 5,4 años. La población de machos representaba el 50,2\% del total, mientras que las hembras representaban el 49,8\%. La proporción de animales que se encontraban esterilizados o castrados fue del $19,2 \%$. Con los resultados encontrados en este estudio las autoridades competentes del distrito serán capaces de optimizar la planificación de las campañas antirrábicas, así como los programas de tenencia responsable de animales de compañía.

PALABRAS CLAVE: Demografía, perros, zoonosis.

\section{SUMMARY}

The aim was to estimate dog demographic characteristics and dog population in the district of Bellavista, Callao. A survey was used for data collection, which was applied at homes selected by a two-stage sampling. On each zone, $20 \%$ of the blocks were selected by a systematic sampling. Then, 10 homes per block were selected. The demographic characteristics of dog population were summarized by descriptive statistic. The study found that $56.1 \%$ of homes owned at least one dog. The average of dogs per home, among homes with dogs, was 1.6. The human: dog ratio was 5,6: 1 . The average age of the dogs was 5.4 years. Male dogs represented $50.2 \%$ of the population, while female represented $49.8 \%$. Sterilized dogs were $19.2 \%$. With these results the authorities could improve the planning of future rabies vaccination campaigns, as well as responsible dog ownership programs.

KEYWORDS: Demography, dogs, zoonoses.

1 Laboratorio de Epidemiología y Salud Pública en Veterinaria, Facultad de Medicina Veterinaria y Zootecnia, Universidad Peruana Cayetano Heredia. Lima, Perú

2 Dirección de Saneamiento Básico, Higiene Alimentaria y Zoonosis de la Dirección Ejecutiva de Salud Ambiental de la Dirección Regional de Salud. Callao, Perú. 


\section{INTRODUCCIÓN}

Los canes son la especie de animales domésticos que se encuentran con mayor frecuencia en las viviendas, compartiendo hábitat con los seres humanos (Hughes \& Macdonald, 2013). Esta interacción entre individuos y canes brinda múltiples beneficios, por ejemplo, en pacientes con enfermedades cardiovasculares, disminuyen la presión arterial y frecuencia cardiaca; mientras que en personas con depresión, aumentan la autoestima y el sentido de responsabilidad (Gómez, Atehortua \& Orozco, 2007). Sin embargo, una tenencia no responsable de estos animales puede generar problemas como la transmisión de enfermedades zoonóticas, accidentes por mordeduras, contaminación, sobrepoblación, etc. (León, Panta, Yarleque \& Falcón, 2013).

Para enfrentar estos problemas se requiere promover y concientizar a la población sobre las necesidades de los animales de compañía y para ello es necesario tener información de la composición de esos animales en la población (Gsell et al., 2012). Para la obtención de esta información es importante la constante actualización y desarrollo de estudios que permitan la obtención de datos sobre la realidad de un área determinada, a fin de invertir en programas preventivos que garanticen la seguridad de las personas y la de los animales de compañía de la zona intervenida (World Health Organization, 2018; Morters et al., 2014).

Los estudios demográficos de la población de canes se realizan en diversas áreas geográficas con la intención de recabar información para futuros programas de control de la población animal y de enfermedades zoonóticas. En el caso del Perú, los primeros estudios en Lima se remontan al año 1976 en donde se estimó la población canina en base a una relación persona: can de 10: 1 para la capital, en el área metropolitana (Málaga, Rodríguez, Inope \& Torres, 1976).

Estudios más actualizados encuentran que esta relación persona: can se ha estrechado. En el distrito de Comas en el año 2013, se estimó una relación de 1 can por cada 5,74 personas (Soriano, Nuñez, León \& Falcón, 2017). En el distrito de San Martín de Porres la relación encontrada fue de 1 can por cada 7 personas (Arauco, Urbina, León y Falcón, 2014). En el año 2016 se realizó un estudio similar en el distrito de Ventanilla - Callao en donde se reportó una relación de 1 can por cada 3,98 personas (Rendón, et al, 2018). Finalmente, en el año 2017 se encontró una relación de 1 can por cada 5 personas en el distrito de San Borja (Arellano, Osorio, Napurí, León, y Falcón, 2017).

Los estudios demográficos en poblaciones de canes permiten tener conocimiento de la realidad de cada región geográfica y favorece la implementación de programas de salud como los de capacitación, prevención y sanidad, con la finalidad de promover condiciones saludables en la población humana y animal. Todo esto llevó a la Municipalidad distrital de Bellavista, Callao, Perú a realizar un estudio de investigación que tuvo como objetivo estimar indicadores demográficos y la población de canes con dueño en su distrito.

\section{MATERIAL Y METODOS}

El estudio se realizó en el distrito de Bellavista, perteneciente a la Provincia Constitucional del Callao - Perú. El análisis de datos se realizó en la Facultad de Medicina Veterinaria y Zootecnia de la Universidad Peruana Cayetano Heredia (FAVEZ-UPCH). El estudio correspondió a una investigación de tipo observacional, transversal, descriptivo.

La Población objetivo del estudio fueron las viviendas pertenecientes al distrito de Bellavista. El tamaño de muestra se determinó usando la fórmula de comprobación de una proporción para poblaciones desconocidas. Las restricciones que se utilizaron fueron: Nivel de confianza del $95 \%$, error máximo admisible del $5 \%$ y proporción esperada del $50 \%$ de viviendas con canes. El tamaño de muestra mínimo calculado fue de 385 encuestas en viviendas.

El instrumento de recolección de información conto con las siguientes variables: Tipo de vivienda (casa, departamento, otros), número de personas por vivienda, tenencia de canes (si o no), número de canes en la vivienda y características demográficas de los canes (sexo, edad, tamaño, raza y si se encontraban esterilizados/castrados o no).

Las viviendas a encuestar se seleccionaron a través de un muestreo bietápico. En la primera etapa se seleccionaron manzanas en los diferentes sectores en los que se encontraba dividido el distrito, posteriormente se seleccionaron 10 viviendas de cada una de las manzanas seleccionadas. La elección de las manzanas y viviendas se realizó siguiendo el modelo de muestreo sistemático. Si no se completaba la muestra con las viviendas de una manzana se tomaba la siguiente manzana hasta completar el número establecido. 
La recolección de encuestas se realizó en conjunto con las autoridades del Centro de Salud Perú Corea, Dirección Regional de Salud de la Región del Callao y con el apoyo de estudiantes de la Facultad de Medicina Humana de la Universidad Nacional Mayor de San Marcos. Todos los encuestadores fueron previamente capacitados en la forma de abordar a los miembros de las viviendas seleccionadas y en el llenado de las encuestas.

La información obtenida en las encuestas fue transferida a una base de datos en el programa Microsoft Excel. Los datos fueron analizados por la herramienta estadística STATA 13.0. Se calculó la proporción de viviendas que poseían canes, la media de canes por vivienda (calculada entre las viviendas que poseían canes) y la relación persona: can. Las variables cualitativas se resumieron mediante tablas de frecuencia.

\section{RESULTADOS}

Se recolectaron 620 encuestas válidas en viviendas del distrito de Bellavista. En el gráfico 1 se muestra la distribución del número de encuestas según zona de muestreo. Dentro del tipo de viviendas el 96,1\% (596) de los encuestados vivía en casas, 3,1\% (19) en departamentos y $0,8 \%$ (5) en otro tipo de vivienda (depósitos y corralones).

En cuanto a la distribución del número de personas por vivienda, se reportó 136 viviendas con cuatro personas $(21,9 \%), 116$ con cinco $(18,7 \%), 94$ con seis
$(15,2 \%)$ y 93 con tres personas $(15,0 \%)$. El total de personas en las viviendas que registró el estudio fue de 3037 personas.

El 56,1\% (348) de las viviendas poseían canes. El promedio de canes por vivienda, entre las viviendas que poseían canes, fue de 1,6. El número de canes por vivienda se distribuyó de la siguiente manera: $65,2 \%$ (227) poseían un can, 20,7\% (72) poseían dos, 8,3\% (29) poseían tres, 4,0\% (14) poseían cuatro, 1,4\% (5) poseían cinco y $0,3 \%$ (1) tenía seis canes en su vivienda, sumando un total de 545 canes. La relación persona: can obtenida fue de 5,6: 1 (3037 personas / 545 perros).

Las características demográficas de los canes que habitaban las viviendas de los encuestados se presentan en la tabla 1. Se observa que la mayoría de los animales eran jóvenes, encontrándose por debajo de los 5 años, la media de edad fue 4,5 años, la moda 2, la mediana 4 años, y los valores extremos estuvieron entre 0,1 a 18 años. Se encontró una proporción similar de animales machos y hembras. En cuanto al tamaño, la mayoría de los encuestados mencionó poseer animales de tamaño pequeño y solo un $19,8 \%$ respondió que su can estaba esterilizado.

De 254 encuestas que mencionaron poseer animales de raza, solamente el 36,6\% (93), especificaron la raza, las cuales se detallan a continuación: Shih Tzu (31), Schnauzer (19), Pequinés (7), Cocker (4), Poodle (4), Beagle (3), Pitbull (3), Golden retriever (2), Jack Russel (2), Labrador (2), Maltes (2), Alaskan Malamute (1),

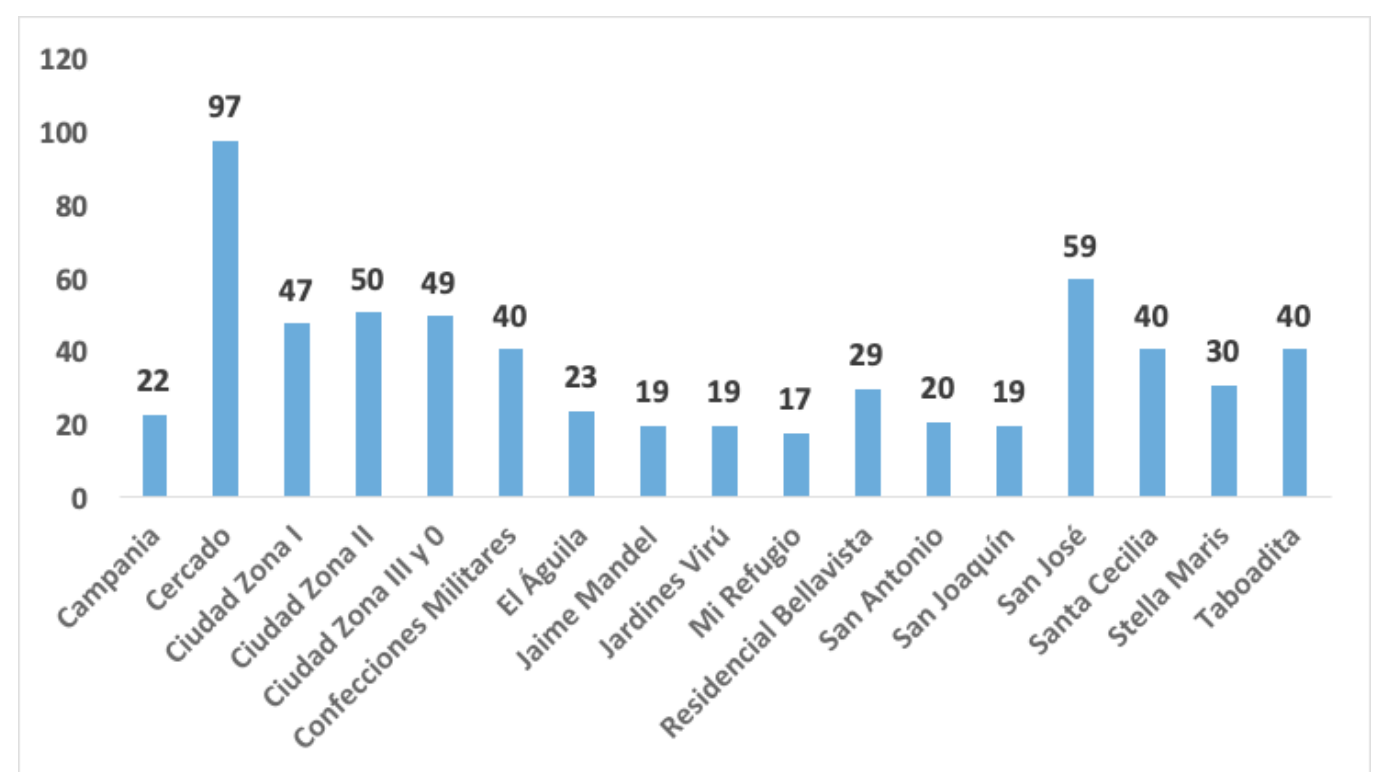

Grafico 1. Distribución del número de encuestas válidas obtenidas en las diferentes zonas del distrito de Ventanilla - Callao, 2017. 
Tabla 1. Características demográficas de los canes en posesión de los entrevistados. Bellavista, Callao. Noviembre 2017.

\begin{tabular}{ccc}
\hline \multicolumn{1}{c}{ Variable } & $\mathrm{n}$ & $\%$ \\
\hline Edad (n $=525)$ & 35 & 6,7 \\
Hasta 0.5 año & 157 & 29,9 \\
$>$ 0.5 a 2 años & 169 & 32,1 \\
$>$ 2 a 5 años & 89 & 17,0 \\
$>$ > a 8 años & 75 & 14,3 \\
$>$ 8 años & & \\
Sexo (n $=524)$ & 263 & 50,2 \\
Macho & 261 & 49,8 \\
Hembra & & \\
Tamaño (n $=526)$ & 258 & 49,0 \\
Pequeño & 172 & 32,7 \\
Mediano & 96 & 18,3 \\
Grande & & \\
Raza (n $=$ 476) & 254 & 53,4 \\
Pura & 222 & 46,6 \\
Cruzada & & \\
Si & 104 & 19,8 \\
No & 420 & 80,2 \\
\hline
\end{tabular}

American bully (1), Bull terrier (1), Chihuahua (1), Chow-Chow (1), Dálmata (1), Doberman (1), Dogo Argentino (1), Fox terrier (1),Galgo Alemán (1),Pastor Alemán (1), Pastor Suizo (1), Perro Peruano sin pelo (1), Siberian Husky (1).

\section{DISCUSIÓN}

El estudio logró obtener un total de 620 encuestas válidas correspondientes a las áreas encuestadas del distrito de Bellavista. Esto representó un 95.4\% del total de encuestas realizadas. El volumen de encuestas realizadas representó el 2,8\% del total de hogares en Bellavista (Compañía Peruana de Estudios de Mercados y Opinión Pública S.A.C [CPI], 2017).

La relación persona: can se suma a otros resultados similares que se han obtenido en Lima y Callao, como el de Soriano et al. (2017) en el distrito de Comas, en donde encontraron una relación persona: can de 5,74: 1, y el resultado de Arellano et al. (2018) en el distrito de San Borja donde se obtuvo una relación de 5:1. Así mismo, difiere de lo hallado por Arauco et al. (2014) en el distrito de San Martín, donde la relación fue de 7.1: 1 y el de Rendón et al. (2018) que encontró una relación de 3,98: 1 en asentamientos humanos del distrito de Ventanilla, Callao.

Algunas de las variaciones en los resultados estarían asociadas a las condiciones socioeconómicas del distrito en estudio. Una mejor economía podría conllevar al cuidado de un mayor número de animales, tal como se refleja en los resultados encontrados en San Borja (Arellano et al., 2018). Sin embargo, un panorama opuesto se puede observar en Ventanilla que presenta una mayor tenencia de canes (Rendón et al., 2017), esto puede deberse a que en este distrito la mayoría de personas pertenece al estrato económico E (CPI, 2017), lo que no permitiría el acceso a servicios veterinarios, entre ellos la esterilización. Esto se reflejaría en una alta tasa de natalidad que mostró el estudio realizado en dicho distrito.

La cantidad de canes sin esterilizar o castrar alcanza niveles elevados por lo que se puede inferir que no existe un cultura de control reproductivo de los canes en estas poblaciones. En San Martin de Porres más del 80\% de canes no se encontraban bajo un programa de control reproductivo (Arauco et al., 2014), valores similares a los encontrados en Bellavista. Se debe de tener en cuenta que la responsabilidad del control reproductivo de los animales de compañía es de los propietarios y las municipalidades solo deberían apoyar este tipo de programas en poblaciones vulnerables que no pueden cubrir el costo del servicio. Un ejemplo de intervención dentro de los parámetros de tenencia responsable de los animales de compañía puede ser la realizada por la municipalidad del distrito de Ventanilla, quien a través de su Clínica Veterinaria Municipal apoyan estas actividades de control reproductivo en Asentamientos Humanos, donde la población carece de recursos para asumir esta responsabilidad (Rojas, 2019).

Los resultados de este estudio y otros realizados en Lima y Callao demuestran que la relación persona: can está por debajo de lo encontrado por Málaga et al. (1976) en donde se halló una relación de 10,5. Esto es importante porque dicha relación todavía es utilizada para planificar la campaña de vacunación antirrábica canina que desarrolla anualmente el Ministerio de Salud a nivel nacional.

Con respecto al porcentaje de hogares con canes, este se encontró dentro de los rangos esperados en zonas urbanas de Lima y Callao. Rendón el al. (2018) encuentran en Ventanilla-Callao que el $61,8 \%$ de viviendas poseían canes; Soriano et al. (2017) reportan $60,4 \%$ de viviendas con canes para el año 2013, en el 
distrito de Ventanilla, Arauco et al. (2014) encuentran $58,2 \%$ en el distrito de San Martín de Porres y Arellano et al. (2018) encontraron que las viviendas con canes correspondían al $54,2 \%$ en el distrito de San Borja. Aparentemente, la condición socioeconómica no estaría afectando la decisión de tener un can.

La promedio de canes, entre las viviendas que poseían canes, fue de 1,6 , esto coincide con lo descrito en el distrito de Comas en el año 2013, donde se encontró un valor similar $(1,74)$ (Soriano et al., 2017). En el distrito de San Martín de Porres se encontró a su vez un promedio de 1,6 (Arauco et al., 2014), en Ventanilla se encontró 1,8 (Rendón et al., 2018), en San Borja 1,4 (Arellano et al., 2018). Estos datos son importantes ya que a mayor cantidad de animales de compañía en casa, habrá mayor exposición a enfermedades zoonóticas; especialmente si no se cumple con los cuidados sanitarios correspondientes.

El tipo de vivienda que predominaba en el lugar de estudio fueron las casas. Los animales se suelen encontrar en mayor número allí, lo que puede estar asociado a la mayor comodidad y facilidad de poseer un can en estos lugares, en comparación con los departamentos. En estos últimos, el espacio, las normas de la junta de propietarios o administración del edificio, o la falta de una persona para la atención de los animales, son los principales obstáculos para tener un can (Arauco et al., 2014).

La proporción de animales machos y hembras, fue similar a los resultados de los estudios realizados en Ventanilla (Rendón et al., 2018), Comas (Soriano et al., 2017), en San Borja (Arellano et al., 2017) y en San Martín de Porres (Arauco et al., 2014). En todos los casos existe mayor población de machos que hembras. Esto puede estar relacionado con la presencia del ciclo estral de las hembras, las gestaciones no deseadas y los consecuentes cuidados de los cachorros. Sin embargo, en otros estudios se señala que el sexo no es una variable importante a considerar para tener un can, debido a que existen métodos de control reproductivos como la esterilización y mayor accesibilidad a servicios veterinarios (Ibarra et al., 2003).

En cuanto al tamaño de los canes, se halló que el tamaño pequeño fue el predominante. Este resultado es similar al hallado en San Borja - Lima, donde el 45,6\% de canes del distrito eran de tamaño pequeño (Arellano et al., 2017). Esto se relacionaría con la mayor facilidad de crianza y manejo que representan los ejemplares pequeños, necesitan menos espacio, su consumo de alimento es menor, y tienen un menor costo de mantenimiento (Arauco et al., 2014). Sin embargo, en San Martín de Porres se encontró resultados distintos, predominio del tamaño mediano con 42,2\% (Arauco et al., 2014), de igual manera en Ventanilla el 40,2\% fueron perros de tamaño mediano (Rendón et al., 2018). Cabe resaltar que en todos los estudios previamente descritos, los canes de tamaño grande se encuentran en menor proporción en la población.

Es particularmente importante la tenencia de animales de raza en Bellavista, lo que difiere a lo encontrado en otros estudios, donde predominó la tenencia de canes mestizos. En San Martín y Comas se reportó que alrededor del $50 \%$ de los canes eran mestizos (Arauco et al., 2014; Soriano et al., 2017), y en Ventanilla se encontró un $80 \%$ de canes mestizos (Rendon et al., 2018). El poseer perros de raza representa una inversión importante a corto y largo plazo, ya que estadísticamente requieren más cuidados médicos (Güttler, 2005; Advocates for Animals, 2006), lo que podría explicar la mayor proporción de canes mestizos. La tenencia de canes de raza pura podría relacionarse también con el estrato socioeconómico alto, esto difiere de la realidad en Bellavista que pertenece a un estrato socioeconómico medio (CPI, 2017). Sin embargo, esto podría ser el resultado de la percepción de las personas sobre sus animales, al creer que son de una raza específica sin conocerla con exactitud y finalmente indicarían que sus animales son de una raza definida cuando en realidad no lo son.

La edad promedio de la población de canes fue de 4,5 años, mostrando una población joven y con capacidad reproductiva, capaz de generar nuevas camadas. Por ello se hace necesario implementar programas que promuevan el control de la reproducción de los canes. En el estudio, solo el 19,8\% de los canes habían sido sometidos a control reproductivo. Valores bajos también se encontraron en el distrito de San Martín de Porres, donde solamente el 11,6\% de canes fueron esterilizados (Arauco et al., 2014) y 9,9\% en Comas (Soriano et al., 2017). La falta de acciones preventivas sumada a una población joven con altos niveles de fertilidad, tendría como resultado un crecimiento acelerado de la población de canes en los próximos años en el distrito (Brusoni, Dezzotti, Fernández y Lara, 2007).

Los resultados obtenidos en el estudio representan un aporte al conocimiento de las poblaciones de canes en zonas urbanas de Perú. Estos serán de utilidad para una adecuada planificación de las campañas de vacunación antirrábica y actividades dentro del marco 
de los programas de tenencia responsable de animales de compañía que las municipalidades deben de cumplir. Particular importancia presenta el control del crecimiento poblacional de canes, ya que tiene impacto en la prevención de enfermedades zoonóticas, y por lo tanto en la salud humana (Fuentes, Pérez, Suárez, Soca \& Martínez, 2006).

\section{CONCLUSIONES}

El estudio "Indicadores demográficos y estimación de la población de canes domésticos en el Distrito de Bellavista, Callao - Perú" llega a las siguientes conclusiones:

- Se estimó que el 56,1\% de los hogares poseían al menos un can en su vivienda.

- El promedio de canes domésticos por vivienda, entre las viviendas que poseían estos animales, fue de 1,6 .

- Se determinó una relación persona: can de 5,6: 1 .

- La edad promedio de la población de canes fue de 5,4 años, con una mediana de 4 años y una moda de 2 años.

- Solamente el 19,8\% de los animales reportados en el estudio se encontraban bajo control reproductivo.

\section{Correspondencia}

Cesar Augusto Harada Gómez

Correo electrónico: cesar.harada@upch.pe

\section{REFERENCIAS BIBLIOGRAFICAS}

1. Advocates for Animals. (2006). The Price of a Pedigree: Dog breed standards and breed-related illness. Edinburgh: Advocates for Animals. Recuperado de: https://onekindplanet.org/uploads/ publications/price-of-a-pedigree.pdf

2. Arauco, D., Urbina, B., León, D., \& Falcón, N. (2014). Indicadores demográficos y estimación de la población de canes con dueño en el distrito de San Martin de Porres, Lima-Perú. Salud tecnol vet, 2(2), 83-92.

3. Arellano, R., Osorio, M., Napurí, MC., León, D., \& Falcón, N. (2018). Indicadores demográficos de perros y gatos con dueño en el distrito de San Borja, Lima-Perú, 2017. Salud Tecnol vet, 6(2), 72-80.

4. Brusoni, C., Dezzotti, A., Fernández, J., \& Lara, J. (2007). Tamaño y estructura de la población canina en San Martín de los Andes (Neuquén). Analecta Veterinaria, 27 (1), 11-23.

5. Compañía Peruana de Estudios de Mercados y Opinión Pública S.A.C. (2017). Perú: Población 2017. Market Report, 7, 9.
6. Fuentes, M., Pérez, L., Suárez, Y., Soca, M. \& Martínez, A. (2006). La zoonosis como ciencia y su impacto social. REDVET, 7 (9), 1-19.

7. Gómez, L., Atehortua, C. \& Orozco, S. (2007). La Influencia de las Mascotas en la Vida Humana. Rev Col de Cienc Pec, 20, 377-386.

8. Gsell, A., Knobel, D., Cleaveland, S., Kazwala, R., Vounatsou, P. \& Zinsstag, J. (2012). Domestic dog demographic structure and dynamics relevant to rabies control planning in urban areas in Africa: the case of Iringa, Tanzania. BMC Veterinary Research, 8, 236.

9. Güttler, V. (2005) Análisis de algunas características de la población canina relacionadas con mordeduras e hidatidosis humana en la provincia de Valdivia. (Tesis de Licenciatura. Universidad Austral de Chile, Valdivia, Chile)

10. Hughes, J. \& Macdonald D.W. (2013) A review of the interactions between free-roaming domestic dogs and wildlife. Biological Conservation, 157, 341-351.

11. Ibarra, L., Morales, M. \& Acuña, P. (2003). Aspectos demográficos de la población de perros y gatos en la Ciudad de Santiago, Chile. Avances en Ciencias Veterinaria, 18, 13-20.

12. León, D., Panta, S., Yarlequé, C., \& Falcón, N. (2013). La convivencia con mascotas en zonas periurbanas: Experiencia en Lima-Perú. MV Rev Cien Vet, 29(4), 21-25.

13. Málaga, H., Rodríguez, J., Inope, L. \& Torres, J. (1976). Epidemiología de la Rabia Canina en Lima Metropolitana. Boletín de la Oficina Sanitaria Panamericana, 1, 405-413.

14. Morters, M., McKinley, T., Restif, O., Conlan, A., Cleaveland, S. \& Hampson, K. (2014). The demography of free-roaming dog populations and applications to disease and population control. Journal of Applied Ecology, 51, 1096-1106.

15. Rendón, D., Quintana, E., Door, I., Vicuña, F., León, D \& Falcón, N. (2018). Parámetros demográficos en la población de canes y gatos domésticos en asentamientos humanos del distrito de Ventanilla, Callao-Perú. Rev investig vet Perú, 29(1), 217-225.

16. Rojas, P., León, D., \& Falcón, N. (2019). Características de los perros y gatos bajo control reproductivo quirúrgico registrados en la Municipalidad de Los Olivos, Lima, Perú. Periodo 2015-2016. Rev Inv Vet Perú, 30(2), 818-827. DOI: http://dx.doi. org/10.15381/rivep.v30i2.16093

17. Soriano, J., Núñez, J., León, D. \& Falcón, N. (2017). Estimación de la población de canes con dueño en el distrito de Comas, Lima - Perú. MV Rev de Cien Vet, 33(2), 5-10.

18. World Health Organization. (2013). Prevention and control of rabies in dogs. En: World Health Organization. Expert Consultation on Rabies. Third report. Switzerland: World Health Organization. 